\title{
Estudio exploratorio de los riesgos a la salud percibidos en población mexicana
}

\author{
Exploratory study of perceived health risks \\ in Mexican population
}

\author{
Esperanza López-Vázquezli, Rosa Lilia Castillo-López² \\ y Zayra García-Chimalpopoca ${ }^{3}$
}

Citación: López V., E., Castillo L., R.L. y García C., Z. (2020). Estudio exploratorio de los riesgos a la salud percibidos en población mexicana. Psicología y Salud, 30(2), 199-206. https://doi. org/10.25009/pys.v30i2.2654.

\section{RESUMEN}

Los riesgos a la salud implican la eventualidad de exponerse a factores que puedan provocar una enfermedad o una lesión, por lo que el objetivo general de este trabajo fue explorar los riesgos a la salud percibidos por participantes mayores de 18 años de la ciudad de Cuernavaca Morelos, un sector de la población. El método utilizado se basó en la técnica de redes semánticas naturales, la cual se aplicó a 220 sujetos. De manera general, los términos de la red pueden dividirse en factores en los cuales el sujeto puede ejercer control y aquellos en los que tal control no existe. Se observaron diferencias en función del sexo, la edad y la escolaridad. En el caso de las mujeres, estas utilizaron el término peligros, mientras que los hombres emplearon el de muerte. El término estrés aparece solo en los grupos de secundaria y preparatoria, mientras que en los de licenciatura y posgrado aparece el de cuidados. Se discute sobre la importancia de las diferencias encontradas para así comprender mejor la forma de pensar de las personas y sus implicaciones en la población sobre la prevención de la salud.

Palabras clave: Riesgo a la salud; Redes semánticas naturales; Significado psicológico; Percepción de riesgo.

\begin{abstract}
The risks to health imply eventually getting exposed to factors that may lead to illness or injury. In this context the general objective of the present study was to explore health risks perceived by the participants. A natural semantic networks technique was applied to 220 participants over 18 years of age from Cuernavaca, Morelos, Mexico. In general, the terms of the network were divided into two factors: those for which the participant can exercise control over the risk factor and those in which he/she cannot. Differences according to sex, age and schooling included: women used the term "dangers" while men used "death." The term stress appeared only in the secondary-preparatory group, while caregiving appeared in the undergraduate-graduate group. The importance of these differences is discussed in the context of better understanding the way of thinking of participating adults and their implications for working on health prevention issues.
\end{abstract}

Keywords: Health; Natural semantic networks; Psychological meaning; Risk perception.

\footnotetext{
${ }^{1}$ Centro de Investigación Transdisciplinar en Psicología de la Universidad Autónoma del Estado de Morelos, Pico de Orizaba 1, Col. Volcanes, 62350 Cuernavaca, Mor., México, tel. (777)329-79-00, correo electrónico: esperanzal@uaem.mx. Artículo recibido el 6 de marzo y aceptado el 13 de septiembre de 2019.

${ }^{2}$ Facultad de Psicología, Universidad Veracruzana, Manantial de San Cristóbal s/n, Col. Xalapa 2000, 91097 Xalapa, Ver., México, tel. (228)842-17-0, correo electrónico: rossy_2502@hotmail.com.

${ }^{3}$ Grupo Ecológico Integral, S.A. de C.V., Av. Centenario 54-148, Int. 4, Col. Civac, 62570 Jiutepec, Mor., México, tel. (777)320-18-70, correo electrónico: zay.garch@gmail.com.
} 


\section{INTRODUCCIÓN}

$\mathrm{L}$

os riesgos a la salud en el presente siglo son el producto de procesos sociales que han provocado diferentes niveles de exposición en términos ambientales, ecológicos y sociales. La Organización Mundial de la Salud [OMS] (2018) alerta sobre los diferentes riesgos actuales, como es el caso de peligros ambientales tales como la contaminación del aire, las altas temperaturas, las infecciones provocadas por el agua insalubre, las inundaciones, los residuos eléctricos y electrónicos mal reciclados y varios más. Por otro lado, entre esos riesgos se encuentran también enfermedades como la diabetes, el cáncer, la enfermedad cerebrovascular, la insuficiencia renal crónica, el consumo de tabaco y alcohol, las dietas poco saludables, el sedentarismo, el sobrepeso y la obesidad, que en los registros de 2018 se presentan como causas importantes en las estadísticas de mortalidad de México (OMS, 2018; Organización Panamericana de la Salud, 2018). Las estimaciones que efectúan estas organizaciones reflejan que la mortalidad, la morbilidad y la discapacidad pueden ser evitadas mediante la reducción de la exposición humana a los peligros y fuentes que pueden provocar dichas enfermedades. Reducir la carga de morbilidad tomando las medidas preventivas necesarias puede generar la mejora de la calidad de vida y el bienestar de las personas, brindándoles así mejores oportunidades de educación y empleo (OMS, 2018).

Hablar de salud no significa referirse únicamente a los aspectos fisiológicos que implica o a la mera ausencia de enfermedad. Se deben considerar con todo rigor, en primera instancia, los aspectos psicológicos, pues ellos influyen profundamente en los comportamientos saludables (Ehrenzweig y Marván, 2018). Dentro de los aspectos psicológicos que intervienen se halla la percepción de riesgo que las personas tienen de las diferentes situaciones y peligros de su entorno y que pueden afectar su salud. Para que tomen las medidas preventivas adecuadas deben percibir primeramente las situaciones de riesgo a las que están expuestas. Es por ello que se considera que en una primera aproximación al trabajo exhaustivo de la preven- ción de la salud está el conocer las situaciones que percibe la población como riesgosas (López y Marván, 2018).

\section{Percepción de riesgos a la salud}

La palabra riesgo se puede definir como la probabilidad o la posible exposición a un peligro o inconveniente que pueda traer consigo una pérdida o un tipo de problema eventual (López y Marván, 2018). Cuando lo anterior se vincula al término salud, se habla de que un riesgo a la salud sería la eventualidad de exponerse a algún factor que pueda provocar una enfermedad o una lesión (OMS, 2018).

La percepción de riesgo es un proceso psicológico que hace posible evaluar la situación amenazante o los riesgos que enfrentan; además, establece una jerarquía de situaciones en las que un peligro puede considerarse de alto riesgo para ciertas personas y de poco para otras. La importancia de conocer la percepción de riesgo es que se esperaría que cuanto mayor sea dicho riesgo percibido, derivado de las diferentes enfermedades o factores potenciales de riesgo para la salud personal, mayor será la motivación que habrá para realizar las acciones protectoras convenientes (Renner, Gamp, Schmälzle y Schupp, 2015).

Desde los años 70 se han incluido los procesos psicológicos y socioambientales en el proceso de salud-enfermedad, toda vez que no se puede ignorar el efecto de lo psicológico, lo emocional, lo social, lo sociodemográfico y lo biológicos en este proceso (Oblitas, 2007). Por ejemplo, respecto a considerar los factores sociodemográficos en la prevención de la salud, un estudio realizado por Ehrenzweig y Marván (2018) con mujeres mexicanas mostró que el comportamiento de prevención hacia el cáncer cervicouterino es muy exiguo entre las mujeres jóvenes y con escasa escolaridad, lo cual las expone a mayor riesgo.

En otro estudio (Dewitt, Davis, Fishhoff y Hanmer, 2017) se demostró que la percepción de riesgos a la salud depende de las preferencias sociales y éticas de los sujetos, por lo que se deben tomar en cuenta los valores de los individuos a quienes tales peligros pueden afectar. Además, se ha 
comprobado que los riesgos percibidos se encuentran influidos por las preferencias y las evaluaciones que el individuo hace al sentirse implicado o amenazado de manera personal por un peligro presente (Renner et al., 2015). También se han estudiado diferentes factores que influyen en la percepción de riesgo, los cuales tienen que ver con la persona y el contexto; por ejemplo si un riesgo es voluntario o involuntario, el conocimiento que se tiene sobre este, la novedad, la gravedad del peligro y el control que se tenga sobre la exposición al mismo. Esta última es una variable que determina el tipo de respuesta del sujeto, pues en función de los recursos con los que cuente y de la capacidad para evitar el peligro es que lo percibirá como controlable o incontrolable (Fishhoff, Slovic, Lichtenstein, Read y Combs, 2000; López y Marván, 2012, 2018).

Por ello, cualquier estudio que abarque de manera integral la investigación en el área de la salud debe considerar siempre la percepción de riesgo de la población involucrada (Catalán, 2006).

Tomando en cuenta que la salud puede estar expuesta a diferentes riesgos que la pueden deteriorar, el objetivo general del presente estudio fue explorar los riesgos y amenazas a la salud percibidos por las personas en la ciudad de Cuernavaca (México). Agregado a ello fue de interés conocer las diferencias entre hombres y mujeres, así como las derivadas de la edad de los participantes y de su nivel de escolaridad. Se optó por aplicar la encuesta a personas mayores de 18 años en virtud de que a esa edad ya se es independiente para decidir la participación, se tiene conciencia del estado de salud y se es ya adulto.

\section{MÉTODO}

\section{Participantes}

La invitación a participar voluntariamente en la investigación estuvo dirigida a mujeres y hombres de entre 18 años y 63 años de edad. Se conformó una muestra no probabilística de 220 participantes, residentes en la mencionada ciudad, que tiene una población aproximada de 366,321 habitantes
(Instituto Nacional de Estadística e Informática [INEGI], 2015). Las encuestas se aplicaron en diferentes colonias, tratando de cubrir la mayor parte de las zonas de la ciudad.

De los participantes, $38 \%$ fueron hombres, con escolaridad de secundaria o preparatoria (43\%) y licenciatura o posgrado $(42 \%)$. El resto no respondió la pregunta sobre su escolaridad y no se incluyó en los análisis.

\section{Instrumento}

El instrumento estuvo conformado por dos secciones: un registro de datos sociodemográficos y una pregunta abierta con la instrucción siguiente: «Cuando escucha la expresión "riesgo para la salud", ¿cuáles son las cinco primeras palabras o imágenes que le vienen a la mente? Después de escribirlas, por favor enumérelas escribiendo al lado izquierdo su orden de importancia». Estas instrucciones están basadas en la técnica de redes semánticas naturales (Figueroa, González y Solís, 1981; Medina, 2004; Reyes, 1993), en la que, además de pedir que se escriban en asociación libre las ideas evocadas por la palabra de interés, se solicita una jerarquización de las ideas relacionadas a esas palabras en la ficha correspondiente.

\section{Procedimiento}

Al momento de recabar los datos se le explicó a cada participante, de manera individual, la dinámica para responder el instrumento y se le presentó un ejemplo para comprobar que la explicación fuera correcta y clara. Para ello, se contó con el apoyo de cinco aplicadores previamente capacitados en el suministro de la encuesta. Se halló a los participantes por solicitud directa haciendo uso de la técnica llamada "bola de nieve". Así, el grupo de encuestadores y una investigadora ubicaron a los participantes en lugares cercanos a sus domicilios, los cuales se encontraban distribuidos en diferentes zonas de la ciudad. La invitación a participar fue de manera individual, y a aquellos que dieron su consentimiento para participar se les informó sobre el anonimato de sus respuestas, tras de lo cual se procedió a aplicarles el instrumento, con 
la opción de responder las preguntas al encuestador o ser contestado por ellos mismos. El tiempo aproximado de aplicación fue de 20 minutos. Después, se les solicitó que llenaran la ficha de datos sociodemográficos y la de redes semánticas.

\section{Análisis de datos}

Para realizar la comparación de los riesgos asociados a la salud manifestados por los participantes se formaron los siguientes grupos: a) sexo (mujeres u. hombres); b) edad (adultos jóvenes, adultos maduros y adultos mayores) y c) escolaridad, donde se buscó tener la mayor homogeneidad (secundaria-preparatoria o licenciatura-posgrado).

El análisis de datos requirió la elaboración de un diccionario en el que se agruparon palabras relacionadas entre sí, de forma que cada grupo se presentó una palabra que fungía como definidora y otra(s) como sinónimo(s) de aquella. Dicho diccionario fue elaborado por tres personas (dos autores y un estudiante), quienes lo revisaron por separado en varias ocasiones hasta que las tres personas estuvieron de acuerdo en que los grupos de palabras (definidora y sinónimos) estaban redactados de forma correcta. Después, se analizaron las ideas asociadas a los riesgos a la salud dados por los participantes según el método establecido por Reyes (1993).

Los términos utilizados en el análisis de las redes semánticas naturales fueron los siguientes:

Tamaño de red (TR): El número total de las diferentes palabras definidoras generadas por los participantes.

Peso semántico (PS): Las primeras diez palabras definidoras de cada participante a las que, de forma conjunta y jerarquizada por frecuencia, se les asigna un valor de forma descendiente de 10 a 1.

Núcleo de red (NR): Las diez definidoras con mayor PS y que representan los elementos que definen al término en interés.

Distancia semántica cuantitativa (DSC): La distancia que hay entre las definidoras que conforman el NR. Se obtiene asignando el 100 por ciento a la palabra definidora con PS más alto; a partir de este valor, se obtuvo la proporción y la diferencia de cada una de las definidoras consecuentes (Reyes, 1993).

\section{Procedimiento}

Primero se calculó el peso semántico de cada palabra definidora utilizada por los participantes de acuerdo a su frecuencia y rango. El peso semántico se obtuvo al analizar las diez palabras que definieron el concepto de interés, de acuerdo a todos los participantes. El número de participantes que mencionaron con mayor frecuencia una palabra se multiplicó por 10; el número de participantes que clasificaron ese mismo término en segundo lugar se multiplicó por 9, y así sucesivamente hasta llegar a 1. Todos estos valores se agregaron, dando como resultado el peso semántico de cada palabra definidora. El siguiente paso fue calcular la distancia semántica cuantitativa entre las palabras definidoras, lo que se hizo mediante el cálculo de proporciones a partir del $100 \%$ que se le otorga a la definidora con mayor peso semántico (peso semántico de la definidora de la que se va a calcular la proporción por 100 , sobre peso semántico de la definidora con mayor peso semántico), y calculando la diferencia entre el 100 y la proporción obtenida con la formula antes mencionada. Finalmente, se obtuvo el núcleo de red, que fue un concentrado de las diez definidoras con mayor peso semántico (Reyes, 1993).

\section{RESULTADOS}

La red semántica de los participantes estuvo compuesta por 269 palabras distintas que, tras ser sometidas al proceso de sinonimia para la construcción del diccionario, disminuyeron a 61, las cuales fueron catalogadas como definidoras.

En el núcleo de red de la muestra general, los participantes definieron "riesgo a la salud" como enfermedades, vicios, malos hábitos, accidentes, contaminación, infecciones, sobrepeso, peligro y muerte, conceptos todos ellos negativos, y un solo elemento positivo: cuidados (Tabla 1).

Al comparar al grupo de mujeres con el de hombres, se observó que ambos grupos tuvieron 
Tabla 1. Distancia semántica cuantitativa $(n=220)$.

\begin{tabular}{|l|c|}
\hline Definidoras & $\mathbf{( \% )}$ \\
\hline Enfermedades & 100 \\
\hline Vicios & 64 \\
\hline Malos hábitos & 55 \\
\hline Accidentes & 37 \\
\hline Contaminación & 31 \\
\hline Infecciones & 28 \\
\hline Sobrepeso & 26 \\
\hline Cuidados & 25 \\
\hline Peligros & 25 \\
\hline Muerte & 24 \\
\hline
\end{tabular}

nueve definidoras en común (enfermedades, malos hábitos, vicios, accidentes, contaminación, infecciones, sobrepeso, cuidados y estrés), sin embargo, el peso semántico que tuvo cada definidora en los grupos fue diferente. Solamente hubo una definidora distinta en cada grupo: las mujeres utilizaron peligros, y los hombres muerte (Tabla 2).

Por otro lado, al realizar la comparación por edad se observó que en los tres grupos la definidora con mayor distancia semántica fue enfermedades (en los tres grupos tiene el cien por ciento de importancia), seguida, también en los tres grupos, de vicios, malos hábitos y accidentes. A pesar de que en los tres grupos de edad se mencionó contaminación, fue en el grupo de adultos maduros donde se posicionó con una mayor distancia semántica (46\%); algo similar se observa con la palabra sobrepeso (48\%). La décima definidora en adultos medios y maduros fue estrés, mientras que en los jóvenes fue peligros (Tabla 3).
Tabla 2. Distancia semántica cuantitativa de los grupos de mujeres y hombres.

\begin{tabular}{|l|c|l|c|}
\hline \multicolumn{2}{|c|}{$\begin{array}{c}\text { Mujeres } \\
\text { (n= 136) }\end{array}$} & \multicolumn{2}{c|}{$\begin{array}{c}\text { Hombres } \\
(\text { n= 84) }\end{array}$} \\
\hline Definidora & D.E. \% & \multicolumn{1}{|c|}{ Definidora } & D.E. \% \\
\hline Enfermedades & 100 & Enfermedades & 100 \\
\hline Vicios & 64 & Malos hábitos & 64 \\
\hline Malos hábitos & 50 & Vicios & 63 \\
\hline Accidentes & 33 & Accidentes & 43 \\
\hline Peligros & 32 & Contaminación & 41 \\
\hline Sobrepeso & 30 & Infecciones & 40 \\
\hline Cuidados & 26 & Muerte & 38 \\
\hline Contaminación & 24 & Cuidados & 24 \\
\hline Estrés & 22 & Sobrepeso & 21 \\
\hline Infecciones & 21 & Estrés & 16 \\
\hline
\end{tabular}

Nota: Las palabras que solo se utilizaron en cada grupo están en cursiva.

En el grupo de participantes que tenían una escolaridad de secundaria-preparatoria y licenciatura-posgrado las definidoras que tuvieron en común y con mayor distancia semántica fueron enfermedades, malos hábitos y vicios. La definidora infecciones ocupó el quinto lugar en el grupo de secundaria-preparatoria, mientras que en el grupo de licenciatura-posgrado se encuentra en el noveno lugar; sin embargo, la distancia semántica fue similar en ambos grupos: 26 y 27\%, respectivamente. Se observó que la definidora estrés aparecía únicamente en el grupo de secundaria-preparatoria, mientras que en el grupo de licenciatura-posgrado era cuidados, introduciendo en este último grupo un elemento positivo (Tabla 4).

Tabla 3. Distancia semántica por edad.

\begin{tabular}{|l|c|l|c|l|c|}
\hline \multicolumn{2}{|c|}{$\begin{array}{c}\text { Adultos jóvenes } \\
\text { (18-30 años) } \\
\text { n= 115 }\end{array}$} & \multicolumn{2}{c|}{$\begin{array}{c}\text { Adultos medios } \\
\text { (31-45 años) } \\
\text { n =68 }\end{array}$} & \multicolumn{2}{c|}{$\begin{array}{c}\text { Adultos maduros } \\
\text { (46 y más) } \\
\text { n= 37 }\end{array}$} \\
\hline Definidoras & D.E. (\%) & Definidoras & D.E. (\%) & Definidoras & D.E. (\%) \\
\hline Enfermedades & 100 & Enfermedades & 100 & Enfermedades & 100 \\
\hline Vicios & 62 & Malos hábitos & 73 & Malos hábitos & 66 \\
\hline Malos hábitos & 41 & Vicios & 71 & Accidentes & 54 \\
\hline Accidentes & 34 & Accidentes & 35 & Vicios & 54 \\
\hline Infecciones & 32 & Contaminación & 28 & Sobrepeso & 48 \\
\hline Contaminación & 28 & Delincuencia & 25 & Peligros & 47 \\
\hline Muerte & 28 & Cuidados & 24 & Contaminación & 46 \\
\hline Sobrepeso & 28 & Muerte & 20 & Infecciones & 37 \\
\hline Cuidados & 27 & Alimentos & 19 & Cuidados & 23 \\
\hline Peligros & 23 & Estrés & 18 & Estrés & 22 \\
\hline
\end{tabular}


Tabla 4. Distancia semántica por escolaridad.

\begin{tabular}{|l|c|l|c|}
\hline $\begin{array}{c}\text { Secundaria-Preparatoria } \\
(\mathbf{n}=\mathbf{9 4})\end{array}$ & \multicolumn{2}{c|}{$\begin{array}{c}\text { Licenciatura-Posgrado } \\
(\mathbf{n}=\mathbf{9 2})\end{array}$} \\
\hline Definidoras & D.E. $\mathbf{( \% )}$ & Definidoras & D.E. (\%) \\
\hline Enfermedades & 100 & Enfermedades & 100 \\
\hline Vicios & 51 & Malos hábitos & 68 \\
\hline Malos hábitos & 41 & Vicios & 63 \\
\hline Accidentes & 32 & Accidentes & 36 \\
\hline Infecciones & 27 & Contaminación & 35 \\
\hline Contaminación & 27 & Cuidados & 33 \\
\hline Peligros & 25 & Sobrepeso & 28 \\
\hline Muerte & 22 & Peligros & 27 \\
\hline Estrés & 21 & Infecciones & 26 \\
\hline Sobrepeso & 19 & Muerte & 24 \\
\hline
\end{tabular}

\section{DISCUSIÓN}

De manera global, y tomando en cuenta las diez definidoras de la red semántica, los resultados muestran que las palabras e ideas asociadas a "riesgo a la salud" están relacionadas con enfermedades, vicios, malos hábitos, accidentes, contaminación, infecciones, sobrepeso, peligro y muerte. Tales definidoras se pueden clasificar claramente en dos tipos: 1) factores internos controlables o de impacto personal, cuyo efecto puede ser dirigido o manipulado por la persona; a estos corresponderían las palabras enfermedades, vicios, infecciones, sobrepeso, malos hábitos y cuidados, y 2) factores externos no controlables, los cuales no dependen directamente de la persona y cuyo control es más difícil; a estos corresponden las palabras accidentes, contaminación, peligro y muerte. La única palabra que se distingue de todas las demás es cuidados, que es la única con connotación positiva al referirse a una acción que podría evitar o disminuir cualquier riesgo a la salud.

Varios estudios han encontrado que uno de los factores de gran peso en la percepción de riesgo es el nivel de control percibido (Fishhoff et al., 2000; Langer y Rodin, 1976; López y Marván, 2012, 2018), y también que este elemento es determinante en la aceptabilidad de un riesgo (López, Brunner y Siegrist, 2012). En un estudio realizado con población expuesta al riesgo volcánico del Popocatépetl, un volcán activo, López y Marván (2012) analizaron la influencia del locus de control, el estrés y las estrategias de afrontamiento que intervienen en dicha población en situación de riesgo constante, hallando que el locus de control externo es un predictor del estilo de afrontamiento pasivo. Ello quiere decir que el locus de control externo causará una respuesta pasiva frente al riesgo volcánico percibido. De acuerdo a dicho estudio, el sentimiento de control es un importante predictor de las acciones del sujeto. En otros estudios se ha observado que las estrategias orientadas a la acción se favorecen cuando los recursos se perciben como suficientes para enfrentar una determinada situación, es decir, que tales recursos permiten controlar la situación estresante o que provoca alguna dificultad al sujeto (Duval y Mulilis, 1999). Se ha encontrado asimismo que el nivel de control percibido influye en la percepción de riesgo en otras investigaciones.

En cuanto a las diferencias entre hombres y mujeres, solamente en la red hacha por las mujeres aparece la palabra peligros, mientras que en la de los hombres aparece muerte. Algunos estudios relacionados con riesgos a la salud han hallado que, en general, las mujeres tienen más identificados los riesgos de contraer diabetes mellitus tipo 2 y muestran además más preocupación que los hombres, lo cual muestra una mayor conciencia del peligro acerca de esta enfermedad (Sánchez, Bonilla, Peña, Costa y Zerquera, 2016). Un estudio realizado por la OMS (2017) mostró que $73 \%$ de todas las defunciones por accidentes de tránsito afectan a varones menores de 25 años, 
quienes tienen tres veces más probabilidades de morir de ese modo que las mujeres jóvenes, por lo que al parecer los hombres tienen más presente la idea de la muerte que las mujeres, sobre todo en México, donde la tasa de dichos accidentes es muy elevada.

Al efectuarse el análisis por grupos de edad se observó que la palabra enfermedades es la que tiene el mayor peso semántico y la que fue jerarquizada en primer lugar por la mayoría de los participantes, lo que indica que el término riesgo a la salud tiene como representación cognitiva primaria la idea relacionada con la enfermedad. De manera lógica, el antónimo de salud es enfermedad, lo que aparece claramente representado en esta primera parte del análisis, toda vez que el riesgo a la salud implicaría la probabilidad de no tenerla. Se observó además que los vocablos contaminación y accidentes tienen mayor distancia semántica entre el grupo de adultos maduros (46\% y 54\%) que entre los más jóvenes, lo que sugiere que dicho grupo manifiesta una mayor preocupación por esos riesgos, los que podría estar determinado por la experiencia, que es uno de los factores que más influyen en la percepción de riesgo (Wachinger, Renn, Begg y Kuhkicke, 2013). En el caso de vicios y sobrepeso, fueron los grupos más jóvenes quienes en mayoría le dieron una posición más alta en la jerarquía.

En la comparación de los datos por grupos de escolaridad, lo que más resalta es el hecho de que solo en el grupo de menor escolaridad (secundaria-preparatoria) aparece la palabra estrés, y que en el grupo de mayor escolaridad (licenciatura-posgrado) únicamente aparece cuidados. Según estos datos, el nivel de estudios establece una diferencia en las ideas asociadas. Si bien muchos participantes del primero de los grupos citados pueden ser adolescentes, no es el caso para todos ellos. Así, se observó una influencia del nivel escolar de los sujetos: aquellos que tienen un menor nivel en su formación consideran riesgo a la salud más como un estresor (Selye, 1978) y hacen una valoración cognitiva de la situación como estresante (Lazarus, 2000). Por otro lado, en el caso de los grupos que tienen mayor nivel de escolaridad la experiencia que han adquirido les permite obtener los recursos intelectuales y las competencias sociales necesarias para enfrentar la vida y las adversidades (Vigotsky, 1934), de manera que cuidados constituye una opción válida para afrontar diferentes riesgos a la salud.

Analizar los elementos que forman parte de los riesgos a la salud percibidos ha permitido conocer las principales preocupaciones de la población bajo estudio. En el presente análisis, se observaron diferencias por género, grupo de edad y nivel de escolaridad, lo cual también muestra que tales variables entrañan diferencias que no se pueden ignorar cuando se quiere trabajar con una población determinada. Investigar la percepción de los riesgos es de gran relevancia debido a que "determina juicios, decisiones, conductas, y conduce a acciones con consecuencias reales" (Pidgeon, 1998, p. 16). Así, este tipo de estudios puede favorecer estrategias de intervención más eficaces en tanto que hacen posible tener un conocimiento más preciso de los conceptos que tienen las personas, lo que favorecerá una mejor comunicación con los participantes, lo que eventualmente se traducirá en el establecimiento de mejores medidas preventivas.

\section{REFERENCIAS}

Catalán V., M. (2006). Estudio de la percepción pública de la contaminación del aire y sus riesgos para la salud: perspectivas teóricas y metodológicas. Revista del Instituto Nacional de Enfermedades Respiratorias, 19(1). Recuperado de http://www. scielo.org.mx/scielo.php?script=sci_arttext\&pid=S0187-75852006000100004.

Dewitt, B., Davis, A., Fishhoff, B. y Hanmer, J. (2017). An approach to reconciling competing ethical principles in aggregating heterogeneous health preferences. Medical Decision Making, 36(6), 1-10.

Duval, T.S. y Mulilis, J.P. (1999). A Person-Relative-to-Event (PrE) approach to negative threat appeals and earthquake preparedness: A field study. Journal of Applied Social Psychology, 29, 495-516.

Ehrenzweig S., Y. y Marván L., M.L. (2018). Behaviour related to cervical cancer risks. En M. L. Marván y E. López V. (Eds.): Preventing health and environmental risks in Latin America (pp. 45-58). London: Springer. 
Figueroa, G., González, E. y Solis, V. (1981). Una aproximación al problema del significado: las redes semánticas. Revista Latinoamericana de Psicología, 13(3), 447-458.

Fishhoff, B., Lichntenstein, S., Read, S. y Combs, B. (1978). How safe is safe enough? A psychometric study of attitudes toward technological risks and benefits. Policy Sciences, 9, 127-152.

Instituto Nacional de Estadística e Informática (2015). Encuesta Intercensal. México: INEGI. Recuperado de http://www.beta. inegi.org.mx/contenidos/proyectos/enchogares/especiales/intercensal/2015/doc/eic_2015_presentacion.pdf.

Langer, E.J. y Rodin, J. (1976). The effects of choice and enhanced personal responsibility for the aged: A field experiment in an institutional setting. Journal of Personality and Social Psychology, 34, 191-198.

Lazarus, R.S. (2000). Estrés y emoción. Manejo e implicaciones en nuestra salud. Bilbao: Desclée de Brower.

López V., E., Brunner, T. y Siegrist, M. (2012). Perceived risks and benefits of nanotechnology applied to the food and packaging sector in Mexico. Brithish Food Journal, 114(2), 197-205.

López V., E. y Marván, M.L. (2012). Volcanic risk perception, locus of control, stress and coping responses of people living near the Popocatépetl volcano in Mexico. Journal of Risk Analysis and Crisis Response, 2(1), 3-12.

López V., E. y Marván, M.L. (2018). Introduction to risk psychology. En M. L. Marván y E. López V.: Preventing health and environmental risks in Latin America (pp. 1-12). London: Springer.

Medina, J.L. (2004). Las redes semánticas naturales, usos y aplicaciones en psicología social (4a ed.). Toluca (México): UAEM.

Oblitas, L. (2007). Enciclopedia de psicología de la salud. Bogotá: PSICOM.

Organización Mundial de la Salud (2017). Lesiones causadas por el tránsito [www Document]. Ginebra: OMS. Recuperado de http://www.who.int/mediacentre/factsheets/fs358/es/.

Organización Mundial de la Salud (2018). Factores de riesgo. Ginebra: OMS. Recuperado de https://www.who.int/topics/risk_ factors/es/.

Organización Panamericana de la Salud (2018). Enfermedades crónicas, factores de riesgo y salud mental. Washington, D.C.: OPS. Recuperado de https:/www.paho.org/mex/index.php?option = com_contentyview $=$ articleyid $=714$ :enfermedades-cronicas-factores-de-riesgo-salud-mentalyItemid $=435$.

Pidgeon, N.F. (1998). Risk assessment, risk values and the social science programme: Why we do need risk perception research. Reliability Engineering and System Safety, 59, 5-15.

Renner, B., Gamp, M., Schmälzle, R. y Schupp, H.T. (2015). Health risk perception. En J. D. Wright (Ed.): International encyclopedia of the social y behavioral sciences (2a ed.) (pp. 702-709). Amsterdam: Elsevier. doi: 10.1016/B978-0-08-0970868.14138-8.

Reyes L., I. (1993). Las redes semánticas naturales: su conceptualización y su utilización en la construcción de instrumentos. Revista de Psicología Social y Personalidad, 11, 81-97.

Sánchez, B., V., Bonilla R., J., Peña V., E., Costa C., M. y Zerquera T., G. (2016). Percepción de riesgo de desarrollar diabetes mellitus en personas no diabéticas. Revista Finlay, 6, 81-92.

Selye, H. (1978). The stress of live. New York: McGraw-Hill.

Vigotsky, L. (1934). Pensamiento y lenguaje. México: Paidós.

Wachinger, G., Renn, O., Begg, Ch. y Kuhkicke, Ch. (2013). The risk perception paradox: Implications for governance and communication of natural hazards. Risk Analysis, 33(6), 1049-1065. 